\title{
Efficacy and Safety of SGLT2 Inhibitors as Adjunctive Treatment in Type 1 Diabetes in a Tertiary Care Center in Saudi Arabia
}

\author{
Walla Fallatah ${ }^{1}$ Imad Brema ${ }^{1} \quad$ Ahmed Alobedallah ${ }^{2}$ Reem Alkhathami ${ }^{1}$ Shawana Zaheer ${ }^{1}$ \\ Eyad AlMalki ${ }^{3}$ Mohammed Almehthel ${ }^{1,4}$ Saad Alzahrani, ${ }^{1,2}$ Mussa H. AlMalki ${ }^{1,2}$ \\ 1 Obesity, Endocrine and Metabolism Center, King Fahad Medical City, \\ Riyadh, Saudi Arabia \\ 2 King Fahad Medical City, College of Medicine, King Saud Bin Abdul \\ Aziz University for Health Science, Riyadh, Saudi Arabia \\ ${ }^{3}$ Colleague of medicine, Shaqra University, Shaqra, Saudi Arabia \\ ${ }^{4}$ Division of Endocrinology, University of British Columbia \\ Address for correspondence Mussa H. AlMalki, MBBS, FACP, FACE, \\ Consultant Endocrinologist, Obesity, Endocrine, and Metabolism \\ Centre, King Fahad Medical City, Riyadh, Saudi Arabia \\ (e-mail: m2malki@yahoo.com).
}

Avicenna J Med 2022;12:10-15.

\author{
Abstract \\ Keywords \\ - type 1 diabetes \\ mellitus \\ - sodium-glucose co- \\ transporter 2 \\ inhibitors \\ - glycemic control \\ - weight gain \\ - diabetic ketoacidosis
}

Background Adjunctive treatment with sodium-glucose co-transporters 2 inhibitors (SGLT2-I) has been successfully used in patients with type 1 diabetes mellitus (T1DM) in recent years to improve glycemic control and reduce body weight without increasing the risk of hypoglycemia; however, there is a scarcity of evidence for real-world experience in their use in T1DM Saudi patients. The purpose of this study was to evaluate the efficacy and safety of empagliflozin as off-label adjunctive therapy in Saudi patients with T1DM.

Methods This study was a retrospective study for T1DM patients, who were prescribed empagliflozin as an adjunctive therapy. Baseline characteristics including age, changes in $\mathrm{HbA1c}$, body weight, total daily insulin dose, lipid profile, and well as side effects such as urinary tract infections (UTIs) and diabetes ketoacidosis (DKA) were evaluated before and after initiation empagliflozin in 37 T1DM patients.

Results The mean age was $25.8 \pm 8.0$ years, mean weight was $75.3 \pm 14.8 \mathrm{~kg}$, mean body mass index (BMI) was $28.1 \pm 6.7 \mathrm{~kg} / \mathrm{m}^{2}$, mean duration of diabetes was $10.1 \pm 6.5$ years, and mean $\mathrm{HbA} 1 \mathrm{c}$ was $9.4 \pm 1.4 \%$. After a mean follow-up duration of $15.8 \pm 6.0$ months, the mean reduction in the $\mathrm{HbA} 1 \mathrm{c} \%$ from baseline was $0.82 \%(p=0.001)$ and mean weight reduction from baseline was $1.7 \mathrm{~kg}(p=0.097)$. The total daily insulin dose was decreased by 2.9 units. UTIs and DKA episodes were reported among $2.7 \%$ and $10.8 \%$ of the participants, respectively.

Conclusion Empagliflozin in combination with insulin in overweight Saudi T1DM subjects resulted in a significant improvement in glycemic control, mild non-significant reduction in body weight, and a small but statistically significant reduction in the total daily insulin dose with a slight increase in the risk of DKA and UTIs. Further larger prospective studies are needed for better evaluation of the efficacy and safety of these agents in Saudi T1DM patients. published online

February 21, 2022
DOI https://doi.org/ 10.1055/s-0041-1742196. ISSN 2231-0770.

\footnotetext{
C 2022. Syrian American Medical Society. All rights reserved. This is an open access article published by Thieme under the terms of the Creative Commons Attribution-NonDerivative-NonCommercial-License, permitting copying and reproduction so long as the original work is given appropriate credit. Contents may not be used for commercial purposes, or adapted, remixed, transformed or built upon. (https://creativecommons.org/ licenses/by-nc-nd/4.0/) Thieme Medical and Scientific Publishers Pvt. Ltd., A-12, 2nd Floor, Sector 2, Noida-201301 UP, India
} 


\section{Introduction}

Type 1 diabetes mellitus (T1DM) is one of the commonest autoimmune diseases affecting children and adolescents worldwide, with an alarmingly rising incidence in Saudi Arabia. ${ }^{1}$ It is estimated to represent $\sim 5$ to $10 \%$ of all cases of diabetes; however, the incidence of T1DM in Saudi Arabia has increased significantly throughout the last three decades. ${ }^{2}$ The estimated total number of children and adolescents under 20 years living with T1DM worldwide in 2019 was 1,101,100 and the estimated number of new cases was nearly 128,900 cases per year. ${ }^{3}$ Moreover, in the Middle East and North Africa (MENA) regions, the estimated total number of children less than 19 years old with T1DM was 149,400 with an annual incidence of 20,800 cases. ${ }^{3}$ Life-long insulin replacement is the mainstay of treatment for T1DM. However, hypoglycemia and weight gain are known side effects of optimal insulin therapy. Therefore, adjunctive treatments (e.g., pramlintide, metformin, incretin analogs) have been proposed to improve glycemic control, reduce glucose variability, reduce the risk of hypoglycemia, and prevent weight gain. ${ }^{4,5}$ Nonetheless, these medications have limitations in T1DM patients when compared with placebo such as the unfavorable side effects profile, short durability, and increased rates of diabetic ketoacidosis (DKA). 6,7

Adjunctive treatment with a more favorable profile would be highly desirable in the management of patients with T1DM. Sodium-glucose co-transporter 2 inhibitors (SGLT2I) are a new class of anti-diabetic medications that have been approved for type 2 diabetes (T2DM) management. SGLT-2 inhibitors prevent glucose and sodium reabsorption from renal tubules and lead to urinary glucose excretion, thereby improving glycemic control without increasing the risk of hypoglycemia. ${ }^{8}$ Moreover, SGLT2-I also promote weight loss, decrease blood pressure, and improve lipid profile. ${ }^{9,10}$ Therefore, the use of SGLT2-I in T1DM may be an option as an offlabel adjunctive oral therapy in addition to insulin for selected patients with T1DM. ${ }^{11,12}$ Several studies have investigated the effect of SGLT2-I in combination with insulin therapy in T1DM and have shown great promising results with positive effects in terms of $\mathrm{HbA} 1 \mathrm{c}$ reduction, prevention of body weight gain, or promotion of weight loss. ${ }^{13-21}$

The aim of this study was to evaluate a real-world clinical experience in the off-label use of empagliflozin in Saudi patients with T1DM.

\section{Objective}

To demonstrate the safety and efficacy of empagliflozin (10 mg and $25 \mathrm{mg}$ doses) as an adjunctive therapy to intensify the effect of insulin in patients with T1DM and assess the changes in HbA1c and weight. Also, we aimed to assess the effect on insulin requirements, blood pressure (BP), lipid profile, and safety.

\section{Materials and Methods}

\section{Patient Selection}

This study was a retrospective chart review of all patients with T1DM at the Obesity, Endocrine and Metabolism Center (OMEC) at King Fahad Medical City (KFMC), Riyadh, who had been prescribed empagliflozin from April 2019 to April 2020. The study was approved by the KFMC Institutional Review Board (IRB); however, informed consent was not obtained as this was a retrospective study. Patients meeting inclusion criteria had a diagnosis of T1DM, were at least 14 years of age or older who received regular care at the adolescents and adult diabetic clinics, were on multiple daily injections consisting of basal insulin and three daily bolus injections, and had received empagliflozin for at least 3 months and had a baseline estimated glomerular filtration rate (eGFR) $>60 \mathrm{~mL} / \mathrm{min}$. In our center as per the Ministry of Health policy, the transition from pediatrics to adults for patients with diabetes starts at age $\geq 14$ years; hence, we included those above 14 years.

\section{Data Collection}

Baseline characteristics were extracted from patient medical records and included age, gender, duration of diabetes (years), type of insulin therapy, HbA1c, weight (kg), body mass index, systolic blood pressure (SBP), diastolic blood pressure (DBP), and lipid profile. The changes in glycemic control and body weight and total daily insulin dose at baseline and the last follow-up visit were evaluated. Safety evaluations of special interest in this study consisted of genital infections, urinary tract infections (UTIs), and DKA was recorded.

DKA was defined by the triad of hyperglycemia, ketosis, and high anion gap metabolic acidosis, whereas the euglycemic DKA included blood glucose level $<250 \mathrm{mg} / \mathrm{dL}{ }^{22}$

\section{Statistical Analysis}

Data entry and statistical analysis were performed using the IBM Statistical Package for Social Sciences (SPSS), version 25. Frequency and percentages were used to describe categorical variables, whereas mean and standard deviation were used to describe quantitative continuous variables. Fischer's exact test was applied to test for the difference between male and female patients regarding categorical variables, whereas student's $t$ test was applied to test for the difference in the means of quantitative continuous variables between male and female patients. Paired $t$-test was applied to compare the means of weight and glycosylated hemoglobin (HbA1c\%), body weight, total insulin dose, and blood pressure before and after empagliflozin intake. Statistical significance was determined at $p<0.05$.

\section{Results}

A total of 37 T1DM patients were included, 22 (59.5\%) were females. Baseline characteristics of patients are listed in -Table 1 . The mean age was $(25.8 \pm 8$ years $)$, the mean HbA1c was $9.4 \pm 1.4 \%$, and the mean duration of diabetes was $10 \pm 6.5$. Comorbidities at baseline were reported in 16 of the patients (43.2\%) and $37.5 \%$ of the participants were obese $\left(\mathrm{BMI} \geq 30.0 \mathrm{~kg} / \mathrm{m}^{2}\right)$ as shown in - Fig. 1 . The mean reduction in the $\mathrm{HbA} 1 \mathrm{c} \%$ was $0.82 \%$ from the baseline to the last followup visit, which was statistically significant $(p=0.001)$. Additionally, the mean weight reduction was $1.7 \mathrm{~kg}$ from baseline to the last clinical visit; however, this did not reach statistical significance $(p=0.097)$. Reduction in both SBP and DBP was 
Table 1 Baseline characteristics of the study population

\begin{tabular}{|l|l|l|l|l|}
\hline Characteristics & Minimum & Maximum & Mean & SD \\
\hline Age, years & 14 & 43 & 25.84 & 8.009 \\
\hline HbA1c $(\%)$ & 6.80 & 13.90 & 9.4 & 1.41469 \\
\hline WT, kg & 54.00 & 112.00 & 75.3143 & 14.85734 \\
\hline BMI $\left(\mathrm{kg} / \mathrm{m}^{2}\right)$ & 21 & 40.7 & 28.1 & 6.7 \\
\hline Systolic BP, $\mathrm{mmHg}$ & 106 & 157 & 125.76 & 13.632 \\
\hline Diastolic BP, mmHg & 37 & 105 & 74.00 & 13.027 \\
\hline Duration of diabetes, years & 1 & 35 & 10.06 & 6.594 \\
\hline Duration of follow-up (months) & 3 & 29 & 15.8 & 6.0 \\
\hline TG, mmol/L (0-1.7) & 0.47 & 2.82 & 1.0738 & 0.53071 \\
\hline Total cholesterol, mmol/L (0-5.18) & 3.70 & 6.40 & 5.0112 & 0.71111 \\
\hline HDL-cholesterol, mmol/L (1-1.5) & 0.00 & 4.50 & 1.4446 & 0.84347 \\
\hline LDL-cholesterol, mmol/L (0-2.6) & 1.70 & 4.07 & 3.1996 & 0.63301 \\
\hline
\end{tabular}

Abbreviations: BMI, body mass index; BP, blood pressure; HbA1c, glycated hemoglobin; HDL: high-density lipoprotein; LDL, low-density lipoprotein; SD, standard deviation; TG, triglyceride; WT, weight.

\section{Co-morbidities}

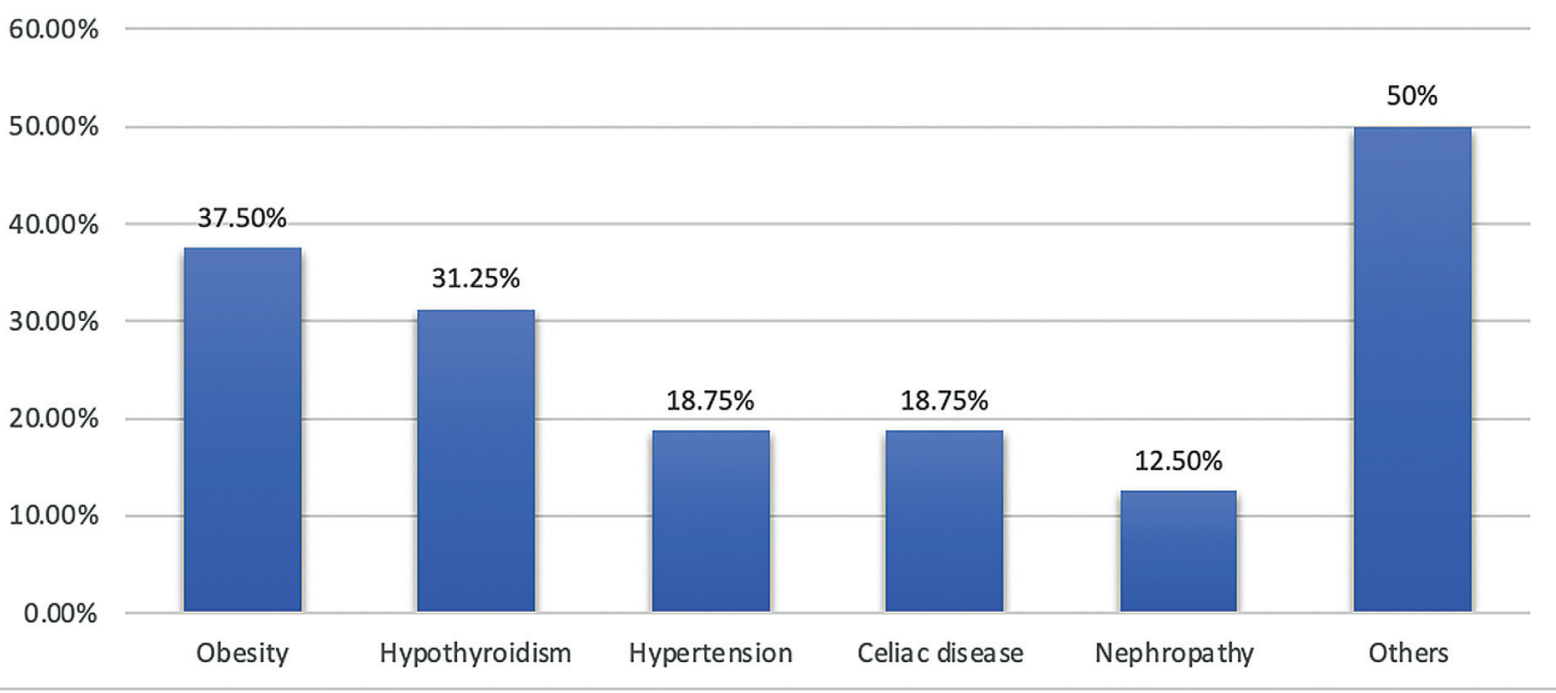

Fig. 1 Co-morbidities among the participants (\%).

not statistically significant. Furthermore, no significant changes were seen in the lipid profile. The total daily insulin dose was reduced from 70.8 units to 67.9 units $(p=0.001)$ with no significant difference between male and female patients (-Table 2). A small subgroup of under 18-yearolds $(N=5)$ with a mean age of 16.2 years had a $0.22 \%$ increase in $\mathrm{HbA} 1 \mathrm{c}$ and a $2.6 \mathrm{~kg}$ reduction in body weight from baseline; however, these values were not statistically significant, as shown in - Table 3.

\section{Adverse Events}

Urinary tract infection was reported in $2.7 \%$ of T1DM patients, and DKA episodes were reported in $10.8 \%$, with no significant difference between male and female patients (-Table 4). Two patients required empagliflozin withdrawal due to mild DKA. For the under 18 subgroups, 2 out of 5 got DKA (40\%) and there were no UTIs (-Table 3 ).

\section{Discussion}

The goal of this study was to assess the safety and efficacy of empagliflozin in Saudi T1DM patients in a real-world practice setting and share our experience with other colleagues following the promising results for the off-label use of this class of anti-hyperglycemic mediations in T1DM patients. To our knowledge, this is the first study to study the use of SGLT2-I in T1DM patients in Saudi Arabia. 
Table 2 Comparisons of parameters before and after adjuvant treatment with empagliflozin

\begin{tabular}{|l|l|l|l|l|}
\hline Biomarker & Pretherapy Mean \pm SD & Posttherapy Mean \pm SD & Mean difference & $p$-Value* \\
\hline HbA1c $(\%)$ & $9.4 \pm 1.4$ & $8.6 \pm 1.6$ & $-0.82 \pm 1.35$ & 0.001 \\
\hline Weight $(\mathrm{kg})$ & $75.3 \pm 15.5$ & $73.6 \pm 15.6$ & $-1.7 \pm 5.88$ & 0.097 \\
\hline Systolic BP (mm Hg) & $125.8 \pm 13.6$ & $125.6 \pm 10.5$ & $-0.21 \pm 10.59$ & 0.910 \\
\hline Diastolic BP (mm Hg) & $74.0 \pm 13.0$ & $74.8 \pm 11.9$ & $+0.82 \pm 13.68$ & 0.728 \\
\hline TG, mmol/L & $1.0 \pm 0.5$ & $1.1 \pm 0.5$ & $+0.07 \pm 0.53$ & 0.529 \\
\hline Cholesterol, mmol/L & $5.0 \pm 0.7$ & $5.0 \pm 0.7$ & $+0.05 \pm 0.65$ & 0.732 \\
\hline HDL, mmol/L & $1.4 \pm 0.8$ & $1.6 \pm 0.8$ & $+0.19 \pm 1.05$ & 0.372 \\
\hline LDL, mmol/L & $3.0 \pm 0.9$ & $3.2 \pm 0.6$ & $-2.9 \pm 5.9$ & $0.17 \pm 0.76$ \\
\hline Total daily dose of insulin (unit) & $70.8 \pm 33.4$ & $67.9 \pm 33.7$ & -278 \\
\hline
\end{tabular}

Abbreviations: BP, Blood pressure; HbA1c, Glycated hemoglobin; HDL, High -density lipoprotein; LDL, Low -density lipoprotein; TG, Triglyceride. *Paired $t$-test.

Table 3 Comparisons of parameters before and after adjuvant treatment with Empagliflozin in under 18 years of age children and adolescents

\begin{tabular}{|l|l|l|l|l|}
\hline Biomarker $\boldsymbol{N = 5}$ & Pretherapy Mean \pm SD & Posttherapy Mean \pm SD & Mean difference & $p_{\text {-Value }}^{*}$ \\
\hline Mean age & $16.2 \pm 0.8$ & - & - & - \\
\hline HbA1c $(\%)$ & $9.5 \pm 0.88$ & $9.7 \pm 1.6$ & $0.22 \pm 1.4$ & 0.75 \\
\hline Weight $(\mathrm{kg})$ & $81.6 \pm 16.9$ & $79.0 \pm 16.1$ & $-2.6 \pm 5.9$ & 0.38 \\
\hline DKA occurrence & $0 / 5$ & $2 / 5(40 \%)$ & - & - \\
\hline UTI occurrence & $0 / 5$ & $0 / 5$ & - & - \\
\hline
\end{tabular}

Abbreviations: DKA, Diabetic Ketoacidosis; HbA1c, Glycated hemoglobin; UTI, Urinary tract infection.

Table 4 Male and female comparisons in the clinical characteristics of the study population at the end of the study

\begin{tabular}{|l|l|l|l|l|}
\hline Patients' characteristics & Total (37) & Males (15) & Females (22) & $p$-Value \\
\hline Duration of diabetes (y) & $10.1 \pm 6.6$ & $9.3 \pm 7.8$ & $10.8 \pm 5.4$ & $0.530^{*}$ \\
\hline Systolic blood pressure, $\mathrm{mm} \mathrm{Hg}$ & $125.6 \pm 10.5$ & $129.8 \pm 12.4$ & $122.6 \pm 8.1$ & $0.049^{*}$ \\
\hline Diastolic blood pressure, $\mathrm{mmHg}$ & $74.8 \pm 11.9$ & $75.7 \pm 14.8$ & $74.2 \pm 9.6$ & $0.720^{*}$ \\
\hline UTI episodes $(n=37)$ No. (\%) & $1(2.7)$ & $0(0.0)$ & $1(5.6)$ & $0.562^{\complement}$ \\
\hline DKA episodes $(n=37)$ No. $(\%)$ & $4(10.8)$ & $1(7.1)$ & $3(15.0)$ & $0.449^{\complement}$ \\
\hline Albumin/Creatinine ratio, & $1.63 \pm 1.40$ & $1.86 \pm 1.8$ & $1.48 \pm 1.1$ & $0.476^{*}$ \\
\hline EGFR $(n=30)$ Mean \pm SD & $125.3 \pm 19.4$ & $124.1 \pm 20.5$ & $125.9 \pm 19.3$ & $0.806^{*}$ \\
\hline Serum creatinine, mmol/L & $58.4 \pm 16.6$ & $68.9 \pm 15.7$ & $51.2 \pm 13.3$ & $0.002^{*}$ \\
\hline Total cholesterol, mmol/L & $5.01 \pm 0.71$ & $4.89 \pm 0.71$ & $5.13 \pm 0.72$ & $0.387^{*}$ \\
\hline HDL-cholesterol, mmol/L & $1.49 \pm 0.48$ & $1.21 \pm 0.27$ & $1.75 \pm 0.5$ & $0.002^{*}$ \\
\hline LDL-cholesterol, mmol/L & $3.2 \pm 0.63$ & $3.34 \pm 0.55$ & $3.07 \pm 0.7$ & $0.289^{*}$ \\
\hline Triglycerides, mmol/L & $1.07 \pm 0.53$ & $1.13 \pm 0.44$ & $1.02 \pm 0.62$ & $0.615^{*}$ \\
\hline
\end{tabular}

Abbreviations: DKA, diabetic ketoacidosis; EGFR, estimated glomerular filtration rate; UTI, urinary tract infection.

Data are presented as mean \pm SD.

*Student's t-test.

ॠischer exact test.

The mean age of our cohort was at around 25 years, mean BMI above $28 \mathrm{~kg} / \mathrm{m},{ }^{2}$ and around $37 \%$ had obesity (BMI $\geq$ $30 \mathrm{~kg} / \mathrm{m}^{2}$ ). The majority of the patients $(86.5 \%)$ of the study participants were adults above 18 years old with a very small number of adolescents (above 14 and under 18 years old) according to our practice, which might be different from other centers.

The present study results have shown a significant reduction in HbA1c of $0.8 \%$ from baseline after mean follow-up duration of treatment of 15 months after adjunctive off-label 
use of empagliflozin. Moreover, there was an average nonsignificant weight reduction of $1.7 \mathrm{~kg}$ but a significant reduction in the total daily insulin dose of $\sim 2.9$ units. However, in the present study, empagliflozin did not significantly reduce both SBP and DBP as well as the lipid profile. These findings are comparable to the findings of other similar previous studies.

The EASE trials were the largest multicenter, placebocontrolled phase 3 trials that have evaluated three doses of empagliflozin $(2.5 \mathrm{mg}, 10 \mathrm{mg}$, and $25 \mathrm{mg}$ ) in over 1,700 subjects with T1DM and demonstrated a placebo-subtracted $\mathrm{HbA} 1 \mathrm{c}$ reduction of $0.28 \%$ for the $2.5 \mathrm{mg}, 0.54 \%$ for the $10 \mathrm{mg}$, and $0.53 \%$ for the $25 \mathrm{mg}^{23}$ Moreover, the EASE trials resulted in a significant mean bodyweight reduction of $-1.8,-3.0$, and $-3.4 \mathrm{~kg}$ for the three consecutive doses, respectively, as well as significant reductions in the total daily insulin doses and systolic blood pressure. ${ }^{23}$ Due to our small sample size we did not separate the $10 \mathrm{mg}$ and $25 \mathrm{mg}$ data; however, our findings are comparable to the EASE trials and our mean duration of follow-up is slightly longer, supporting the durability of the effect of empagliflozin as an adjunctive treatment for T1DM, which has been questioned. However, the rate of DKA in our study was relatively higher at around $10.8 \%$ compared with much lower rates of $4.3 \%$ and $3.3 \%$ for the $10 \mathrm{mg}$ and $25 \mathrm{mg}$ doses, respectively, in the EASE trials. The rate of UTIs was comparable between our study and most of other studies at 3.1\%.

In a meta-analysis from China that was published in 2017 and included T1DM patients aged between 18 and 65 years old, the addition of SGLT2-I to insulin decreased fasting blood glucose and total insulin dosage as compared with placebo ${ }^{24}$.

Furthermore, in a randomized controlled trial from Japan published in 2018, empagliflozin led to a dose-dependent significant increase in 24-hour urinary glucose excretion (UGE) compared with placebo after 7 days of treatment and after 4 weeks of treatment. The UGE increase was associated with improved glycemic control, reduction in body weight, and insulin needs ${ }^{25}$. Additionally, empagliflozin treatment resulted in a dose-dependent increase in serum ketone bodies and free fatty acids.

In a study from Italy that was published in 2019 and involved a meta-analysis of RCTs, sotagliflozin (which is unlike empaglflozin, is both SGLT-1 and 2 inhibitors approved for use as adjunctive treatment in T1DM patients in Europe) reduced HbA1c, fasting plasma glucose, 2-hour postprandial plasma glucose, total daily insulin dose, basal, and bolus insulin doses. ${ }^{26}$ Moreover, there was an increase in time in range and improvement of other continuous glucose monitoring parameters. In addition, sotagliflozin reduced body weight, systolic blood pressure, and albuminuria. More recently, a study from Spain published in 2019, with a followup of 52 weeks, showed that empagliflozin in addition to optimization of insulin therapy in T1DM patients, significantly decreased HbA1c, total daily insulin requirements, body weight, and systolic blood pressure. ${ }^{27}$

In addition to the previous evidence in support of the benefits of SGLT2-I as adjunctive therapy in T1DM, a systematic review and meta-analysis done by Chen et al in 2017 demonstrated that the addition of empagliflozin to daily multiple insulin doses in type 1 diabetes significantly reduced fasting plasma glucose, glycosylated hemoglobin A1C (by $0.37 \%$ ), body weight (by $2.54 \mathrm{~kg}$ ), and total daily insulin dose (by $6.22 \mathrm{IU}$ ) as compared with the control group. ${ }^{28}$

A comparison between the aforementioned studies, including the present one, should be interpreted with caution, considering differences in the inclusion criteria, particularly the duration of insulin treatment and baseline $\mathrm{HbA} 1 \mathrm{c} \%$ level as well as dosage and regimen of empagliflozin.

In the present study, UTI episodes and DKA episodes were reported among $2.7 \%$ and $10.8 \%$ of T1DM patients, respectively, with no gender difference, and all DKAs were hyperglycemic, unlike most other studies in which euglycemic DKA occurrence was more frequent. Our finding showed that the DKA rate was consistent with what was reported previously in patients without the use of SGLT-2 inhibitors in Saudi Arabia with a DKA frequency ranging from $25-80 \%{ }^{29}$

Although the present study represents a real-world clinical experience of patients with T1DM prescribed empagliflozin in diabetes clinics in Saudi Arabia, it has several important limitations such as the small sample size, the retrospective study design, and lack of a comparator arm. We also acknowledge other limitations such as the fact that it is from a single-center experience, which will reduce the generalizability of our results. Moreover, the inclusion of under 18 years old adolescents and pediatric subjects might confound our results and make comparisons more difficult with other similar studies done in other parts of the world.

\section{Conclusion}

Our study shows that empagliflozin in combination with insulin therapy in mostly overweight Saudi T1DM subjects significantly reduced HbA1c by $0.8 \%$ over an average followup period that is slightly longer than that reported in most previous studies with additional benefits of mild non-significant reduction of body weight and a significant reduction in the total daily dose of insulin. DKA risk remains a major side effect and a limitation for adjunctive therapy with this class of anti-diabetic medications and further multicenter prospective placebo-controlled studies for relatively longer durations are still needed to focus on finding the smallest, safest dose that gives the glycemic and weight control benefits while avoiding serious side effects such as DKA.

\section{Authors' contributions}

All authors contributed to the conception, planning, and conduct of the study, and to drafting and revising of the manuscript and approval of its final version.

\section{Compliance with Ethical Principles}

This study was approved by the Institutional Review Board of King Fahad Medical City, Riyadh, Saudi Arabia.

\section{Consent}

The study participants were not consented as the IRB waive consent due to the nature of the study. 
Sponsorship and Funding

The study received no specific sponsorship or funding in any shape of form.

\section{Conflicts of Interest}

None declared.

\section{References}

1 Robert AA, Al-Dawish A, Mujammami M, Dawish MAA. Type 1 diabetes mellitus in Saudi Arabia: a soaring epidemic. Int J Pediatr 2018;2018:9408370. Doi: 10.1155/2018/9408370

2 Al-Herbish AS, El-Mouzan MI, Al-Salloum AA, Al-Qurachi MM, AlOmar AA. Prevalence of type 1 diabetes mellitus in Saudi Arabian children and adolescents. Saudi Med J 2008;29(09):1285-1288

3 Patterson C, Karuranga S, Salpea P, Saeedi P, Dahlquist G, Soltesz G, et al. Worldwide estimates of incidence, prevalence and mortality of type 1 diabetes in children and adolescents: results from the International Diabetes Federation Diabetes Atlas, 9th edition. Diabetes Res Clin Pract (in press). Doi: 10.1016/j.diabres.2019.107842

4 Gubitosi-Klug RA, Braffett BH, White NH, et al; Diabetes Control and Complications Trial (DCCT)/Epidemiology of Diabetes Interventions and Complications (EDIC) Research Group. Risk of severe hypoglycemia in type 1 diabetes over 30 years of follow-up in the DCCT/EDIC study. Diabetes Care 2017;40(08):1010-1016[PMC free article][PubMed] [Google Scholar]

5 Mathieu C, Gillard P, Benhalima K. Insulin analogues in type 1 diabetes mellitus: getting better all the time. Nat Rev Endocrinol 2017;13(07):385-399[PubMed] [Google Scholar]

6 Al Khalifah RA, Alnhdi A, Alghar H, Alanazi M, Florez ID. The effect of adding metformin to insulin therapy for type 1 diabetes mellitus children: a systematic review and meta-analysis. Pediatr Diabetes 2017;18(07):664-673[PubMed] [Google Scholar]

7 Mathieu C, Zinman B, Hemmingsson JU, et al; ADJUNCT ONE Investigators. Efficacy and safety of liraglutide added to insulin treatment in type 1 diabetes: the ADJUNCT ONE treat-to-target randomized trial. Diabetes Care 2016;39(10):1702-1710 [PubMed] [Google Scholar]

8 Ferrannini E, Solini A. SGLT2 inhibition in diabetes mellitus: rationale and clinical prospects. Nat Rev Endocrinol 2012;8 (08):495-502

9 Baker WL, Smyth LR, Riche DM, Bourret EM, Chamberlin KW, White WB. Effects of sodium-glucose co-transporter 2 inhibitors on blood pressure: a systematic review and meta-analysis. J Am Soc Hypertens 2014;8(04):262-275

10 Monami M, Nardini C, Mannucci E. Efficacy and safety of sodium glucose co-transport-2 inhibitors in type 2 diabetes: a metaanalysis of randomized clinical trials. Diabetes Obes Metab 2014; 16(05):457-466[PubMed] [Google Scholar]

11 Dashora U, Patel D, Gregory R, Winocour P, Dhatariya K, Nagi D. Association of British Clinical Diabetologists (ABCD) position statement on the use of sodium-glucose cotransporter-2 inhibitors in type 1 diabetes (updated 2019). Br J Diabetes 2019;19:66-72. Doi: 10.15277//bjd.2019.208[CrossRef] [Google Scholar]

12 Danne T, Garg S, Peters AL, et al. International consensus on risk management of diabetic ketoacidosis in patients with type 1 diabetes treated with sodium-glucose cotransporter (SGLT) inhibitors. Diabetes Care 2019;42(06):1147-1154. Doi: 10.2337/dc182316[PMC free article] [PubMed] [CrossRef] [Google Scholar]

13 Oelze M, Kröller-Schön S, Welschof P, et al. The sodium-glucose co-transporter 2 inhibitor empagliflozin improves diabetes-induced vascular dysfunction in the streptozotocin diabetes rat model by interfering with oxidative stress and glucotoxicity. PLoS
One 2014;9(11):e112394. Doi: 10.1371/journal.pone.0112394 [PMC free article] [PubMed] [CrossRef] [Google Scholar]

14 Cheng ST, Chen L, Li SY, Mayoux E, Leung PS. The effects of empagliflozin, an sglt2 inhibitor, on pancreatic $\beta$-cell mass and glucose homeostasis in type 1 diabetes. PLoS One 2016;11(01): e0147391. Doi: 10.1371/journal.pone.0147391[PMC free article] [PubMed] [CrossRef] [Google Scholar]

15 Henry RR, Rosenstock J, Edelman S, et al. Exploring the potential of the SGLT2 inhibitor dapagliflozin in type 1 diabetes: a randomized, double-blind, placebo-controlled pilot study. Diabetes Care 2015;38(03):412-419

16 Sands AT, Zambrowicz BP, Rosenstock J, et al. Sotagliflozin, a dual SGLT1 and SGLT2 inhibitor, as adjunct therapy to insulin in type 1 diabetes. Diabetes Care 2015;38(07):1181-1188

17 Henry RR, Thakkar P, Tong C, Polidori D, Alba M. Efficacy and safety of canagliflozin, a sodium-glucose cotransporter 2 inhibitor, as add-on to insulin in patients with type 1 diabetes. Diabetes Care 2015;38(12):2258-2265[PubMed] [Google Scholar]

18 Pieber TR, Famulla S, Eilbracht J, et al. Empagliflozin as adjunct to insulin in patients with type 1 diabetes: a 4 -week, randomized, placebo-controlled trial (EASE-1). Diabetes Obes Metab 2015;17 (10):928-935

19 Cherney DZ, Perkins BA, Soleymanlou N, et al. Renal hemodynamic effect of sodium-glucose cotransporter 2 inhibition in patients with type 1 diabetes mellitus. Circulation 2014;129 (05):587-597

20 Cherney DZ, Perkins BA, Soleymanlou N, et al. The effect of empagliflozin on arterial stiffness and heart rate variability in subjects with uncomplicated type 1 diabetes mellitus. Cardiovasc Diabetol 2014;13:28[PMC free article] [PubMed] [Google Scholar]

21 Perkins BA, Cherney DZ, Partridge H, et al. Sodium-glucose cotransporter 2 inhibition and glycemic control in type 1 diabetes: results of an 8-week open-label proof-of-concept trial. Diabetes Care 2014;37(05):1480-1483

22 Barski L, Eshkoli T, Brandstaetter E, Jotkowitz A. Euglycemic diabetic ketoacidosis. Eur J Intern Med 2019;63:9-14. Doi: 10.1016/j.ejim.2019.03.014

23 Rosenstock J, Marquard J, Laffel LM, et al. Empagliflozin as adjunctive to Insulin Therapy in Type 1 Diabetes: The EASE Trials. Diabetes Care 2018;41(12):2560-2569. Doi: 10.2337/dc18-1749

24 Yang Y, Pan H, Wang B, Chen S, Zhu H. Efficacy and safety of SGLT2 inhibitors in patients with Type 1 Diabetes: A meta-analysis of randomized controlled trials. Chin Med Sci J 2017;32(01):22-27

25 Shimada A, Hanafusa T, Yasui A, et al. Empagliflozin as adjunct to insulin in Japanese participants with type 1 diabetes: Results of a 4-week, double-blind, randomized, placebo-controlled phase 2 trial. Diabetes Obes Metab 2018;20(09):2190-2199. Doi: 10.1111/dom. 13351

26 Musso G, Gambino R, Cassader M, Paschetta E. Efficacy and safety of dual SGLT 1/2 inhibitor sotagliflozin in type 1 diabetes: metaanalysis of randomised controlled trials. 2019;365(11328)1-16. Doi: $10.1136 /$ bmj.11328

27 Bayona Cebada A, Nattero-Chávez L, Alonso Díaz S, EscobarMorreale HF, Luque-Ramírez M. Efficacy and safety of SGLT2 inhibitors in type 1 diabetes after the introduction of an off-label use protocol for clinical practice. Diabetes Technol Ther 2020;22 (03):208-215

28 Chen J, Fan F, Wang JY, et al. The efficacy and safety of SGLT2 inhibitors for adjunctive treatment of type 1 diabetes: a systematic review and meta-analysis. Sci Rep 2017;7:44128. Doi: $10.1038 /$ srep 44128

29 Zayed H. Epidemiology of diabetic ketoacidosis in Arab patients with type 1 diabetes: a systematic review. Int J Clin Pract 2016;70 (03):186-195. Doi: 10.1111/ijcp.12777 